\title{
Comparison of FT-NIR Transmission and HPLC to Assay Montelukast in Its Pharmaceutical Tablets
}

\author{
Ahmed B. Eldin ${ }^{1 *}$, Abdalla A. Shalaby ${ }^{2}$ \\ ${ }^{1}$ Sigma Pharmaceutical Corp., Egypt \\ ${ }^{2}$ Analytical Chemistry Department, Faculty of Pharmacy, Zagazig University, Zagazig, Egypt \\ E-mail:a.badr@sigma-pharm.com \\ Received February 25, 2011; revised April 6, 2011; accepted April 25, 2011
}

\begin{abstract}
For several years, near-infrared spectroscopy (NIRS) has become an analytical technique of great interest for the pharmaceutical industry, particularly for the non-destructive analysis of dosage forms. The goal of this study is to show the capacity of this new technique to assay the active ingredient in low-dosage tablets. NIR spectroscopy is a rapid, non-destructive technique and does not need any sample preparation. A prediction model was built by using a partial least square regression fit method. The NIR assay was performed by transmission. The results obtained by NIR spectroscopy were compared with the conventional HPLC method for Montelukast tablets produced by Sigma pharmaceutical corp. The study showed that Montelukast tablets can be individually analyzed by NIR with high accuracy. It was shown that the variability of this new technique is less important than that of the conventional method which is the HPLC with UV detection.
\end{abstract}

Keywords: FT-NIR Transmission, PAT, Validation, HPLC Assay, Montelukast Tablets, PLS Model

\section{Introduction}

Currently the Food and Drug Administration adopt what is known as process analytical technology (PAT) initiative which is a collaboration effort with industry to facilitate the introduction of new and efficient manufacturing technologies. PAT are systems for design, analysis, and control of manufacturing processes, based on timely measurements of critical quality and performance attributes of raw and in-process materials and products, to assure high quality of products at the completion of manufacturing (http://www.fda.gov/cder) [1]. PAT includes scientifically based process design that identifies key measurements of product quality and the critical process variables that affect them, appropriate measurement devices, statistical information technology tools, and feedback process control strategies that work together to ensure production of final products with the desired quality.

Several vibrational spectroscopy techniques are used for the application of PAT in the on-line monitoring of the pharmaceutical process. For several years, near-infrared spectroscopy (NIRS) has become an analytical technique of great interest for the pharmaceutical Industry. NIR spectroscopy is a rapid, non-destructive tech- nique and requires none or minimal sample pretreatment. The NIR region spans the wavelength range 12,500 $4000 \mathrm{~cm}^{-1}$.

In this region, absorption bands correspond mainly to overtones and combinations of fundamental vibrations [2].

In the pharmaceutical sector, several qualitative and quantitative applications of NIR spectroscopy have been described during manufacturing steps. In the beginning of manufacturing process, NIR can be used for the identification of active substances and excipients [3-5]. By recording a NIR spectrum, it has been shown that identity, crystallinity, and water content are controlled making NIRS an interesting tool for the characterization of raw materials. The blending step can also be followed by NIRS [6]. It is well known that creating homogeneous blend is one of the most important step during manufacturing of most of dosage forms in pharmaceutical Industries. Typically, the most time consuming part of the blending process is not the blending itself but the analysis that must be performed to validate the final homogeneity of the drug substance in the blend especially in low strength preparation. In practice the relationship between concentration and absorbance is empirically determined by calibration. In the first step, spectra of substances with 
known composition are recorded. Then, these acquired spectra and the data available from a reference analysis are used to determine a calibration function. In the second step, spectra of substances with unknown composition are measured and then used to determine the properties of interest by means of the calibration function $[7,8]$. Processing NIR data can be carried out in a number of ways to simplify the spectral information. It has been shown that data pretreatment could be a key step for success of NIR spectroscopy [9].

The aim of the study is to show the agreement between the NIR technique and the HPLC-UV detection assay method.

\section{Experimental}

\subsection{Materials and Methods}

\subsubsection{Materials}

All materials were kindly supplied by Sigma pharmaceuticals Corp. - Egypt. The commercial samples of Montelukast tablets were used and a placebo contains the same raw materials used in the production process, were used. The placebo was used to make serials of dilutions for establishing the calibration curve. All materials are of pharmaceutical grade and include microcrystalline cellulose, magnesium stearate, Croscarmellose sodium and Aerosil 200 are kindly supplied by Sigma pharmaceuticals Corp., to be of the same type used for preparation of Montelukast tablets.

\subsubsection{Analytical Procedures}

NIR Spectroscopy

FT-NIR Spectrometer MPA Flexible NIR spectrometer from Bruker Optics (Germany) [See Figure 1], for method development and quality control.

Optional extension modules are available for connecting up to 2 fiber optic probes, NIR probe for liquids "quartz". Pistol grip model with external trigger and LED status lights. Includes $2 \mathrm{~m}$ fiber optic cable with Bruker Quick connect. Fixed optical path length of $1 \mathrm{~mm}$. Solid Probe has a head of $80 \mathrm{~mm}$ length and utilizes an integrating sphere for analysis of solid samples in diffuse reflectance and a measurement unit for analyzing highly scattering solid media in transmission.

The spectrometer is equipped with a fast, PC-based data system with OPUS/IR FT-IR Spectroscopy Software Package Version 5.0 which was provided by Bruker Optics. OPUS IDENT is a software package designed to identify substances by their NIR spectra while OPUS Quant is designed for the quantitative analysis. For this purpose, QUANT used a partial least square (PLS) fit

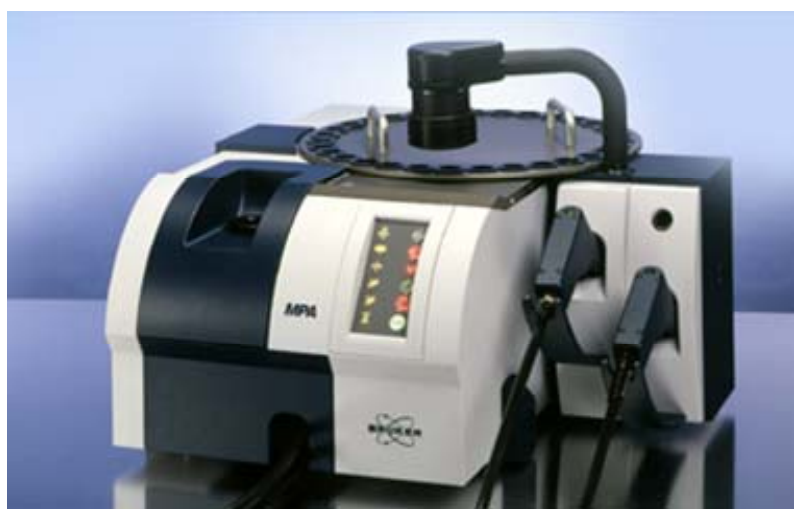

Figure 1. FT-NIR Spectrometer MPA flexible NIR spectrometer.

method. In PLS, the calibration involves correlating the data in the spectral matrix $X$ with the data in the concentration matrix $Y$.

This means that the factoring of the spectral data is more suited for concentration prediction. Construction of the PLS model: in a first step a PLS regression model was built using calibration samples. The obtained model was chemometrically validated by leave-one-out cross validation. The final PLS model was described by a selected spectral region, a certain spectra pretreatment and a number of PLS factors. To build the model, eleven different concentrations of tablets were prepared and 5 samples were measured per concentration. To obtain these different concentrations, raw materials and placebo are used and samples were prepared on Lab. scale. Each spectrum was the average of 32 scans and the spectrophotometer was operated at a resolution of $8 \mathrm{~cm}^{-1}$.

Spectral data pretreatments: NIR spectra are affected by the state of the analyzed material. The baseline can drift and maximum absorbance may change. Spectral pretreatments correct these interferences $[10,11]$. In our study, a normalisation and a first derivative were used to enhance spectral information and to reduce baseline drift. The normalisation method used was a vector normalisation. The absorbance of the Montelukast tablets samples was measured and NIR spectrums were saved. Measurements were performed by the NIR fiber optic probe for solids. Before measurement a measurement for the background must be taken and the detector single shall be checked. In developing method the measurement conditions shall be determined and saved to be recalled in each measurement time to avoid result variation and ensure high accuracy of the developed analytical procedure. Samples were measured in amber colored glass samples and minimum illumination in the measurement place were kept to ensure that there was no stray light during measurement

The measurement time for each sample was about 10 
seconds per scan and the instrument was operated at a resolution of $8 \mathrm{~cm}^{-1}$ between 4000 and $12,000 \mathrm{~cm}^{-1}$. In first trials five scans for each sample were taken and a mean spectrum for them was calculated but it was found that no difference between the mean spectrum and the single one that is due to the complete homogeneity of the measured sample.

\subsubsection{Reference Method}

Simple, sensitive and accurate stability indicating analytical method for Montelukast has been used by using RP-HPLC techniques and applying the proposed method in the assay of Montelukast pure material, tablets and the brand product (sigulair ${ }^{\mathbb{R}}$ ), since there is no official monograph. Chromatography was performed with mobile phase containing a mixture of acetonitrile and $0.01 \mathrm{M}$ potassium dihydrogen phosphate buffer, $\mathrm{pH} 4.0(7: 3, \mathrm{v} / \mathrm{v})$ with flow rate of $1.0 \mathrm{ml}$ per min., C18 column and UV detection at $355 \mathrm{~nm}$. developed method satisfies the system suitability criteria, peak integrity, and resolution for the parent drug and its degradants. The method was validated for linearity (correlation coefficient $=0.9999$ ), accuracy, robustness and precision.

\section{Results and Discussions}

\subsection{Methods Validation}

Figure 2 shows the raw spectra obtained with the calibration samples.

A vector normalization was applied to these spectra.

From these spectra, three regions were selected, automatically by the Quant program; it was between 11,250 and $8500 \mathrm{~cm}^{-1}$.

All samples were also analyzed by the HPLC method. The following validation items were applied for the reference HPLC method.

\subsubsection{Linearity}

The linearity of calibration curves (peak area vs. concentration) for Montelukast in pure solutions as well as in the drug-matrix solutions were checked over the concen- tration ranges of about 50,100,150,200, 250 and 300 $\mu \mathrm{g} / \mathrm{ml}$, each concentration level was injected 3 times $(\mathrm{n}=$ 3 ) and the average peak area was calculated. The resulting curve was found to be linear with correlation coefficients of better than 0.999 in most cases (Table 1). Furthermore, Table 1 lists the linearity parameters of the calibration curves for Montelukast in pure and drug-matrix solutions.

\subsubsection{Sensitivity and accuracy}

The limits of detection LOD and limits of quantitation, LOQ, were calculated for the calibration graphs of Montelukast as three and ten times of the noise level for LOD and LOQ, respectively [USP-32; 2009]. The values for LOD and LQD are given in Table 1. The accuracy of an analytical method is the closeness of test results obtained by that method to the true value [USP-32; 2009]. The accuracy of the method was tested by analyzing different samples of Montelukast at various concentration levels ranged from $150-250 \mu \mathrm{g} / \mathrm{ml}$ in either pure solutions or in solutions comprising the drug-matrix used in tablet formulation, each concentration level was injected 3 times $(n=3)$ and the average peak area was calculated. The results were expressed as percent recoveries of the Montelukast in the samples (Table 2). Table 2 shows that the overall percent recoveries of Montelukast in pure and drug-matrix solutions were $100.11 \%$, relative standard

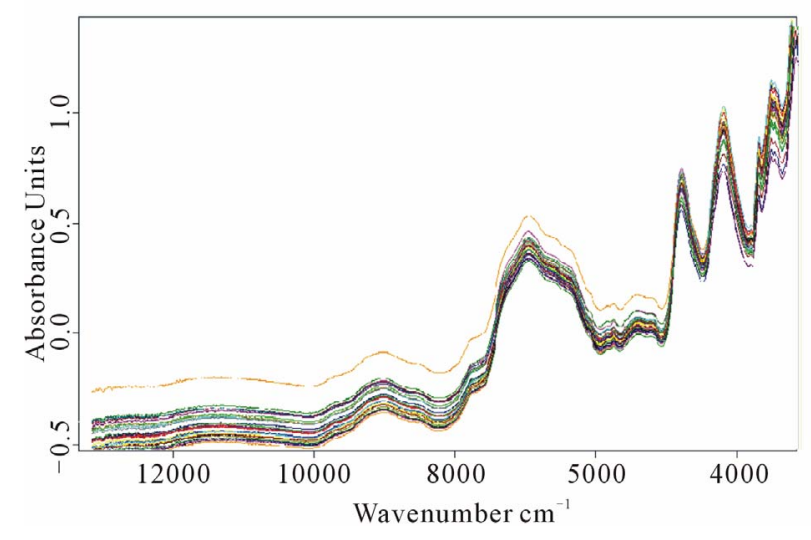

Figure 2. The FT-NIR spectrum for Montelukast tablets.

Table 1. Linearity of calibration curve for Montelukast in standard preparations and in drug-matrix preparation. Number of points in the regression line is 6 for each case.

\begin{tabular}{|c|c|c|c|c|c|c|c|c|}
\hline Item & $\begin{array}{c}\text { Calibration } \\
\text { range }(\mathrm{mcg} / \mathrm{ml})\end{array}$ & $\begin{array}{l}\text { Correlation } \\
\text { coefficient }\end{array}$ & Slope & $\begin{array}{c}\text { Slope } 95 \% \\
\text { confidence interval } \\
\text { for the slope }\end{array}$ & Intercept & $\begin{array}{c}\text { Slope } 95 \% \\
\text { confidence interval } \\
\text { or the intercept }{ }^{\mathrm{a}}\end{array}$ & $\begin{array}{l}\text { LOD } \\
\mathrm{mcg} / \mathrm{ml}\end{array}$ & $\begin{array}{c}\mathrm{LOQ} \\
\mathrm{mcg} / \mathrm{m}\end{array}$ \\
\hline $\begin{array}{c}\text { Montelukast in } \\
\text { standard preparation }\end{array}$ & $50-300$ & 0.9999 & 0.690 & \pm 0.0110 & -0.125 & \pm 1.985 & 5.51 & 9.36 \\
\hline $\begin{array}{c}\text { Montelukast in } \\
\text { drug-matrix preparation }\end{array}$ & $50-300$ & 0.9999 & 0.685 & \pm 0.0180 & 1.023 & \pm 2.987 & 6.24 & 15.42 \\
\hline
\end{tabular}

Confidence intervals of the slope and the intercept $=($ S.D of the slope or intercept $\mathrm{x} t)$, the value of $t$ at 3 degree of freedom and $95 \%$ confidence level is 3.18 . 
deviation (R.S.D.) $=0.35 \%$ and $99.9 \%$ (R.S.D. $)=5.27 \%$, respectively.

\subsubsection{Stability of Analytical Solutions}

Sample solutions of Montelukast in pure and drug-matrix solutions were tested for HPLC stability over $24 \mathrm{~h}$. The samples were analyzed by the optimized HPLC method in fresh and stored solutions. The percent difference observed was in the range of -0.31 to -0.76 (Table 3 ), indicating the possibility of using standard solutions of Montelukast in pure or drug-matrix solutions over a period of $24 \mathrm{~h}$ without degradation.

\subsubsection{Precision}

As stated in the ICH [12] and FDA [13] guidelines, the precision of an analytical procedure expresses the closeness of agreement (degree of scatter) between a series of measurements obtained from multiple samplings of the same homogeneous sample under prescribed conditions. In this study precision was evaluated through repeatability and reproducibility.

Various samples containing about $200 \mathrm{mcg} / \mathrm{ml} \mathrm{Mon-}$ telukast in a synthetic matrix (drug-matrix) were analyzed by three independent analysts (six samples each) over 1 day and various days.

The 1 day repeatability gave the overall percent recoveries of $100.1 \%, 101.2 \%$ and $99.9 \%$ with $\%$ R.S.D. of $1.2,0.62$ and 0.26 , respectively. The long-term reproducibility for all the analysis gave an over all recovery and R.S.D. of $100.4 \%$ and $0.96 \%$, respectively.

\subsubsection{Robustness}

As defined by the $\mathrm{ICH}$, the robustness of an analytical procedure refers to its capability to remain unaffected by small and deliberate variations in method parameters $[14,15]$. In order to study the simultaneous variation of

Table 2. Estimation of the accuracy as an item for validation of the proposed HPLC method for the determination of Montelukast in standard or drug matrix solution.

\begin{tabular}{|c|c|c|c|c|}
\hline \multirow{2}{*}{ Quantity added in $\mathrm{mcg} / \mathrm{ml}$ of Montelukast } & \multicolumn{2}{|c|}{ Standard solutions } & \multicolumn{2}{|c|}{ Drug-matrix solutions } \\
\hline & Quantity found in $\mathrm{mcg} / \mathrm{ml}$ & Recovery (\%) & Quantity found in $\mathrm{mcg} / \mathrm{ml}$ & Recovery (\%) \\
\hline 150.2 & 150.4 & 100.1332 & 151.2 & 152.1 \\
\hline 161.3 & 160.9 & 99.75201 & 165.7 & 164.8 \\
\hline 172.4 & 173 & 100.348 & 175.4 & 173.9 \\
\hline 182.3 & 183.1 & 100.4388 & 184.1 & 184 \\
\hline 193.7 & 192.9 & 99.58699 & 195.1 & 194.4 \\
\hline 203.1 & 202.7 & 99.80305 & 205.1 & 204.4 \\
\hline 221.2 & 222.2 & 100.4521 & 219.8 & 220.2 \\
\hline 253.4 & 254.4 & 100.3946 & 250.7 & 252.2 \\
\hline \multirow{2}{*}{ Calculations: } & Average & $100.11 \%$ & Average & $99.90 \%$ \\
\hline & $\%$ R.S.D & $0.350 \%$ & $\%$ R.S.D & $0.527 \%$ \\
\hline
\end{tabular}

Table 3. Stability of Montelukast in standard and drug-matrix solutions over a period of 24 ha.

\begin{tabular}{|c|c|c|c|c|}
\hline \multirow{2}{*}{ Sample } & \multirow{2}{*}{ Quantity added in $\mathrm{mcg} / \mathrm{ml}$ of Montelukast } & \multicolumn{2}{|c|}{ Quantity found } & \multirow{2}{*}{ Difference $(\%)$} \\
\hline & & Fresh solution & Stored solution & \\
\hline \multirow{3}{*}{$\begin{array}{l}\text { Standard solution } \\
\text { of Montelukast. }\end{array}$} & 100.18 & 100.19 & 100.21 & -0.14 \\
\hline & 201.89 & 200.95 & 200.9 & 0.16 \\
\hline & 302.76 & 301.5 & 301.55 & -0.07 \\
\hline \multirow{3}{*}{$\begin{array}{l}\text { Standard solution } \\
\text { of Montelukast in } \\
\text { the drug-matrix. }\end{array}$} & 100.18 & 99.8 & 100.13 & 0.49 \\
\hline & 201.89 & 200.87 & 200.53 & 1.07 \\
\hline & 302.76 & 301.65 & 300.49 & 0.22 \\
\hline
\end{tabular}

${ }^{\mathrm{a}}$ Difference $(\%)=($ Quantity found in fresh solution - Quantity found in stored solution $) /($ Quantity found in fresh solution $) \times 100$. 
the factors on the considered responses, a multivariate approach using design of experiments is recommended in robustness testing. A response surface method was carried out to obtain more information and to investigate the behavior of the response around the nominal values of the factors. Response surface methodology (RSM) has the following advantages: (a) to allow a complete study where all interaction effects are estimated; (b) to give an accurate description of an experimental region around a center of interest with validity of interpolation [15]. Generally the large numbers of experiments required by standard designs applied in RSM discourage their use in the validation procedure. However, if an analytical method is fast and requires the testing of a few factors (three or less), a good choice for robustness testing may be the central composite design (CCD), widely employed because of its high efficiency with respect to the number of runs required. A CCD in $\mathrm{k}$ factors requires $2 \mathrm{k}$ factorial runs, $2 \mathrm{k}$ axial experiments, symmetrically spaced at $\alpha \pm$ along each variable axis, and at least one center point [17]. Two to five center repetitions are generally carried out in order to know the experimental error variance and to test the predictive validity of the model [18]. In order to study the variables at no more than three levels $(-1,0$, +1 ), the design used in robustness testing of montilukast was a central composite design (CCD) with $\alpha= \pm 1$. Three factors were considered: percentage $\mathrm{v} / \mathrm{v}$ of acetonitrile (x1); flow rate $\mathrm{ml} \cdot \mathrm{min}^{-1}(\mathrm{x} 2)$ and $\mathrm{pH}(\mathrm{x} 3)$. The experimental domain of the selected variables is reported in Table 4. The ranges examined were small deviations from the method settings and the corresponding responses in the peak area considered (Y) were observed. A three-factor CCD requires 9 experiments, including two replicates of the center point. The experimental plan and the corresponding responses are reported in Table 4. All experiments were performed in randomized order to minimize the effects of uncontrolled factors that may introduce a bias on the response. A classical seconddegree model with a cubic experimental domain was postulated. Experimental results were computed by Minitab-15. The coefficients of the second-order polynomial model were estimated by the least squares regression. The regression equation was as follow:

$$
\mathrm{AUC}=127933+221 \text { AcN\% } \% 27628 \text { F.R }-555 \mathrm{pH}
$$

The factor flow rate (x2) was significant for the regression model assumed.

The model was validated by the analysis of variance (ANOVA). The statistical analysis showed (Table 5) that the model represents the phenomenon quite well and the variation of the response was correctly related to the variation of the factors, Figure 3 shows the influence of each of the variables studied in the montelukast as a response where none of them exceeds the limit except the
Table 4. Experimental domain of the selected variables.

\begin{tabular}{|c|c|c|c|c|}
\hline Exp. No. & $\mathrm{AcN} \%$ & F.R & $\mathrm{pH}$ & AUC \\
\hline 1 & 63 & 0.8 & 3.5 & 119297 \\
\hline 2 & 77 & 0.8 & 3.5 & 120962 \\
\hline 3 & 63 & 1.2 & 3.5 & 108200 \\
\hline 4 & 77 & 1.2 & 3.5 & 102096 \\
\hline 5 & 63 & 0.8 & 4.5 & 118187 \\
\hline 6 & 77 & 0.8 & 4.5 & 118742 \\
\hline 7 & 63 & 1.2 & 4.5 & 103206 \\
\hline 8 & 77 & 1.2 & 4.5 & 106535 \\
\hline 9 & 63 & 0.8 & 4 & 105980 \\
\hline 10 & 77 & 1.2 & 4 & 110974 \\
\hline 11 & 70 & 1 & 4 & 108200 \\
\hline 12 & 70 & 1 & 4 & 117078 \\
\hline 13 & 70 & 1 & 3.5 & 118742 \\
\hline 14 & 70 & 1 & 4.5 & 119852 \\
\hline 15 & 70 & 1 & 4 & 119852 \\
\hline 16 & 70 & 1 & 4 & 119297 \\
\hline
\end{tabular}

Table 5. Analysis of variance for the experimental plan and the corresponding responses.

\begin{tabular}{cccccc}
\hline Source & DF & SS & MS & F & P \\
\hline Regression & 3 & 295839577 & 98613192 & 2.95 & 0.076 \\
Residual & 4 & 401025227 & 33418769 & & \\
Total & 7 & 696864804 & & & \\
\hline
\end{tabular}

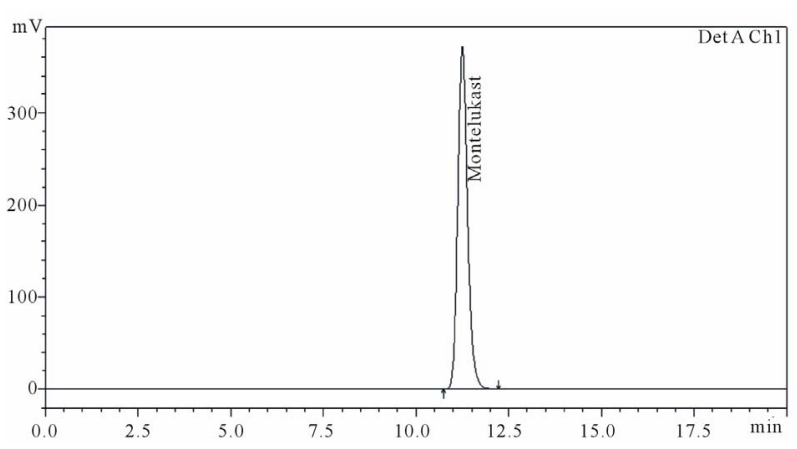

Figure 3. The resulting chromatogram for montelukast.

flow rate as clear. The interpretation of the results has to start from the analysis of the whole model equation rather than from the analysis of the single coefficients. It is important for the response surface study, to consider also the factors whose coefficients are statistically non 
significant. For this reason the analysis of the response surface plot is necessary. As shown in Figure 3 the analysis produces three-dimensional graphs by plotting the response model against two of the factors, while the third is held constant at a specified level, usually the proposed optimum. Figure 4 shows a graphical representation of the isoresponse surface for variation of percentage of

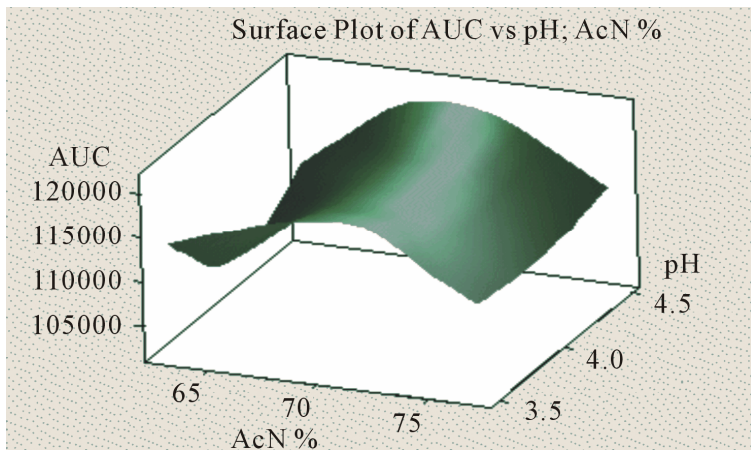

(a)

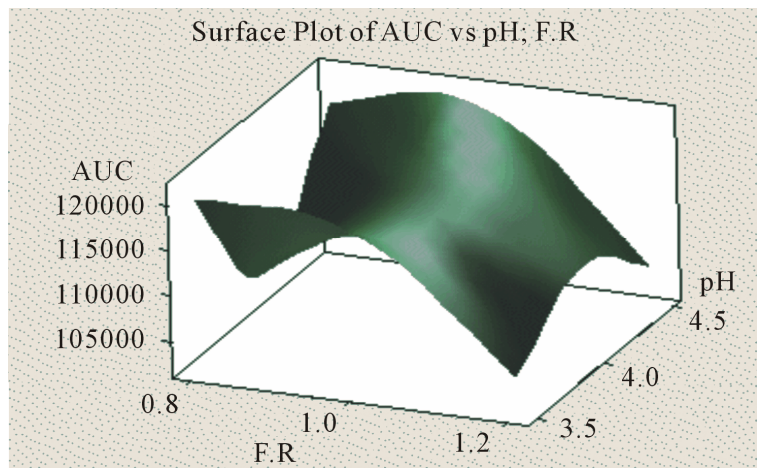

(b)

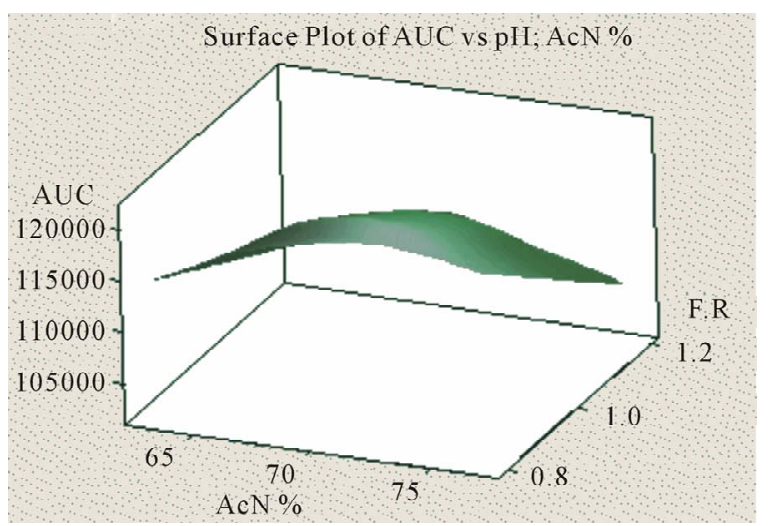

(c)

Figure 4. Three-dimensional plot of the response surface for $Y$ (found drug peak area ratio). (a) Variation of the response $Y$ as a function of $x 1$ (\% acetonitrile) and $x 2$ (flow rate); fixed factor: $x 3(\mathrm{pH})=3.0$; (b) Variation of the response $Y$ as a function of $x 1(\%$ Acetonitrile) and $x 3(\mathrm{pH})$ fixed factor: $x 2$ (flow rate) $=1.0 \mathrm{ml} \cdot \mathrm{min}^{-1}$; (c) Variation of the response $Y$ as a function of $x 2$ (flow rate) and $x 3(\mathrm{pH})$; fixed factor: $x 1(\%$ acetonitrile $)=50 \% \mathrm{v} / \mathrm{v}$.
$\mathrm{ACN}(\mathrm{x} 1)$ and flow rate (x2), while the $\mathrm{pH}(\mathrm{x} 3)$ is maintained constant at its optimum of 4.0. An increase in the flow rate results in a decrease of the observed peak area ratio $(\mathrm{Y})$, while the percentage of organic modifier had no important effect on the response. Analogous interprettation may be derived by examining the factors flow rate (x2) versus $\mathrm{pH}(\mathrm{x} 3)$, where the factor flow rate is maintained constant and the method can be considered robust for the studied experimental response. In conclusion, by examining the ANOVA results and analysis of response surface confirms that $\mathrm{Y}$ is not robust for factor $\mathrm{x} 2$, thus a precautionary statement should be included in the analytical procedure for this factor.

\subsubsection{Predictability}

To evaluate the predictability of the model, the relative standard error of prediction (RSEP) was used [16].

$$
\operatorname{RSEP}(\%)=\sqrt{\frac{\sum_{i=1}^{n}\left(C_{H P L C}-C_{N I R}\right)}{\sum_{i=1}^{n} C_{H P L C}}}
$$

where $C$ is the amount of Montelukast as measured by the HPLC reference method and the NIR method and $n$ is the number of samples.

The chosen model has a RMSECV value of $1.76 \%$.

This regression model gave a coefficient of correlation (r2) of 99.48 .

This regression, which indicate the relation between the predicted and true values is shown in Figure 5.

\subsubsection{Agreement between the Two Methods for Unknown Samples}

According to Bland and Altman's method, the first step is to examine the data. A simple plot of the results given by a method versus those of the other one is a useful start. However, the data points will usually be clustered near the line and it will be difficult to assess between method differences so that a plot of the difference between the methods against their mean is chosen. This plot of data may be more informative. Figure 6 shows the distribu-

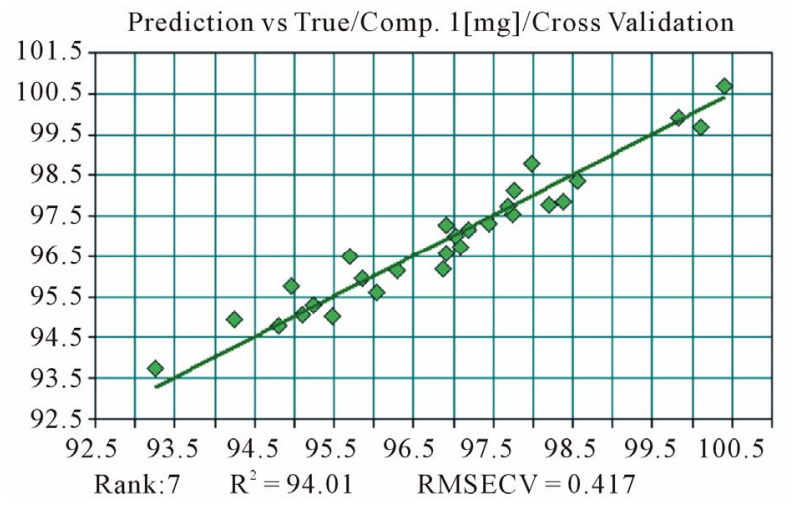

Figure 5. Regression of the calibration samples. 


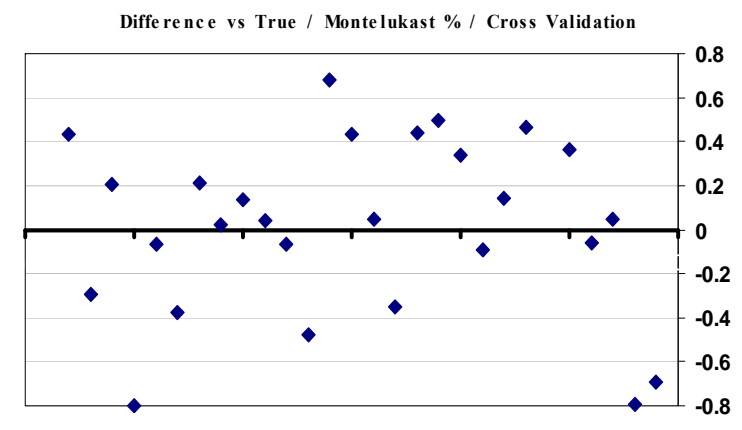

Figure 6. Distribution of the differences against their mean.

tion of the differences against their mean.

\section{Conclusions}

NIR spectroscopy has been shown to be a viable alternative to HPLC with UV detection for the assay of Montelukast tablets, and it takes only few minutes to analyse a batch once the calibration model has been set up. The proposed model is easy to use and give accurate results. It is a non-destructive method and thus lends itself very well for on-line/at-line production control purposes.

Compared to the conventional technique, the NIR spectroscopy method is faster, non-destructive, and gives less variability. It has been shown that NIRspectroscopy can replace safely the UV-vis spectrophotometry.

\section{Acknowledgements}

The authors thank Sigma Pharmaceutical Corp., Egypt for technical support.

\section{References}

[1] FDA, "PAT - A Framework for Innovative Pharmaceutical Manufacturing and Quality Assurance," 2004. http://fda.gov/cder

[2] E. W. Ciurczak and J. K. Drennen, "Practical Spectroscopy Series: Pharmaceutical d Medical Applications of Near-Infrared Spectroscopy," Marcel Dekker, New York, 2002.

[3] W. Plugge and C. Van der Vlies, "The Use of Near Infrared Spectroscopy in the Quality Control Laboratory of the Pharmaceutical Industry," Journal of Pharmaceutical and Biomedical Analysis, Vol. 10, No. 10-12, 1992, pp. 797-803. doi:10.1016/0731-7085(91)80083-L

[4] C. I. Gerh"ausser and K. A. Kovar, "Strategies for Constructing Near-Infrared Spectral Libraries for the Identification of Drug Substances," Applied Spectroscopy, Vol. 51, No. 10, 1997, pp. 1504-1510. doi:10.1366/0003702971939000

[5] M. J. Vredenbregt, P. W. J. Caspers, R. Hoogerbrugge and D. M. Barends, "Choice and Validation of a Near
Infrared Spectroscopic Application for the Identity Control of Starting Materials.: Practical Experience with the EU Draft Note for Guidance on the Use of Near Infrared Spectroscopy by the Pharmaceutical Industry and the Data to be Forwarded in Part II of the Dossier for a Marketing Authorization," European Journal of Pharmaceutics and Biopharmaceutics, Vol. 56, No. 3, 2003, pp. 489-499. doi:10.1016/S0939-6411(03)00119-X

[6] S. S. Sekulic, H. W. Ward and P. K. Aldridge, "On-Line Monitoring of Powder Blend Homogeneity by Near-Infrared Spectroscopy," Analytical Chemistry, Vol. 68, No. 3, 1996, pp. 509-513. doi:10.1021/ac950964m

[7] P. Merckle and K.-A. Kovar, "Assay of Effervescent Tablets by Near-Infrared Spectroscopy in Transmittance and Reflectance Mode: Acetylsalicylic Acid in Mono and Combination Formulations," Journal of Pharmaceutical and Biomedical Analysis, Vol. 17, No. 3, 1998, pp. 365374. doi:10.1016/S0731-7085(97)00194-5

[8] R. P. Cogdill, C. A. Anderson and J. K. Drennen, Pharmaceutical Technology, 2004, pp. 29-34.

[9] J. Sun, Journal of Chemometrics, Vol. 11, 1997, pp. 525-532.

[10] R. J. Barnes, M. S. Dhanoa and S. J. Lister, "Standard Normal Variate Transformation and De-trending of NearInfrared Diffuse Reflectance Spectra," Applied Spectroscopy, Vol. 43, No. 5, 1989, pp. 772-777. doi:10.1366/0003702894202201

[11] T. Fearn, NIR News, Vol. 10, 1999, pp. 10-11.

[12] ICH Q2B, International Conference on Harmonisation, Validation of Analytical Procedures, Methodology, 2002.

[13] FDA, "Guidance for Industry: Validation of Analytical Procedures," Food and Drug Administration, Rockville, 1997.

[14] International Conference on Harmonisation Topic Q2B, "Validation of Analytical Methods: Methodology," The Third International Conference on Harmonization of Technical Requirements for Registration of Pharmaceuticals for Human Use (ICH) Yokohama-Japan.

[15] Y. V. Heyden, A. Nijhuis, J. Smeyers-Verbeke and B. G. M. Vandeginste D. L., Journal of Pharmaceutical and Biomedical Analysis, 2001, p. 24723.

[16] A. Eustaquio, P. Graham, R. D. Jee, A. C. Moffat and A. D. Trafford, "Quantification of Paracetamol in Intact Tablets Using Near-Infrared Transmittance Spectroscopy," Analyst, Vol. 123, No. 11, 1998, pp. 2303-2306. doi: $10.1039 / \mathrm{a} 804528 \mathrm{c}$

[17] R. Ragonese, M. Mulholland and J. Kalman, "Full and Fractionated Experimental Designs for Robustness Testing in the High-Performance Liquid Chromatographic Analysis of Codeine Phosphate, Pseudoephedrine Hydrochloride and Chlorpheniramine Maleate in a Pharmaceutical Preparation," Journal of Chromatography A, Vol. 870 , No. 1-2, 2000, p. 45. doi:10.1016/S0021-9673(99)00972-3

[18] G. A. Lewis, D. Mathieu and R. Phan-Tan-Luu, "Pharmaceutical Experimental Design," Marcel Dekker, New York, 1999. 